\section{Current and evolving knowledge of prognostic factors for pediatric ependymomas}

Felipe Andreiuolo, ${ }^{1,2}$, Céline Ferreira', Stéphanie Puget ${ }^{1,3}$ \& Jacques Grill*1,4

${ }^{1}$ Centre National de la Recherche Scientifique Unité Mixte de Recherche 8203 'Vectorology \& Anticancer Therapeutics', Gustave Roussy Cancer Institute, Paris-Sud University, Villejuif, France

2Department of Pathology, Gustave Roussy Cancer Institute, Paris-Sud University, Villejuif, France

${ }^{3}$ Department of Neurosurgery, Necker Sick Children's Hospital, Paris V Descartes University, Paris, France 4Department of Pediatric \& Adolescent Oncology, Gustave Roussy Cancer Institute, Paris-Sud University, Villejuif, France

*Author for correspondence: Tel.: +33 142116209 - Fax: +33 142115275 - grill@igr.fr

Ependymomas are one of the most common pediatric malignant brain tumors. Prognosis, especially in young children, remains poor due to their inherent chemoand radio-resistance and effective treatment remains one of the more difficult tasks in pediatric oncology: up to half of the patients may die from the disease. The only reproducible prognostic factor is the extent of surgery; neither histological grading nor other biomarkers can be used to reliably make treatment decisions in clinical practice. None of the studies identifying new biomarkers have been conducted prospectively, only few have been undertaken within the context of a clinical trial and most have been conducted with limited samples (often including adults and childhood samples). International collaboration is needed to improve ependymoma prognostication.

The annual incidence of adult and pediatric ependymomas, in western countries is two cases per million. Ependymoma is the third most common brain tumor in children after astrocytomas and medulloblastomas. It can be located anywhere in the CNS, but is predominantly found in the posterior fossa in children, and the spine and supratentorial regions in adults. Increasing age (adults vs children), spinal location and complete resection are associated with improved survival. There has not been a significant therapeutic change in the last 20 years. Treatment is mainly based on surgery with or without radiation therapy. There is an urgent need for prognostic markers to tailor the treatment strategies.

\section{Definitions}

Virchow was the first to describe ependymal tumors in 1863-1865 [1]. In 1899, Störch described the perivascular pseudorosettes and ependymal rosettes, which are still major histological criteria for the diagnosis of ependymoma [2]. The relation of ependymomas to normal ependyma was further stressed [3], based on the identification of cytoplasmic structures derived from centrioles named blepharoplasts, or basal bodies, also present in normal ependyma, which form the base for cilia and flagella that extend out of the cell [4]. Interestingly, very recent studies have shown particular subsets of ependymoma with upregulation of genes involved in ciliogenesis [5] and the overexpression of proteins associated with centrioles at the time of recurrence in most ependymomas [6]. Ependymomas and ependymoblastomas were set apart as distinct entities by Bailey and Cushing in 1926, and, since then, there has been controversy regarding their relationship and even the real existence of the latter [1,7]: ependymoblastomas are currently included among embryonal tumors in the WHO 2007 classification, while ependymomas are included among neuroepithelial lesions. Roussy and Oberling distinguished ependymocytomas "ependymoma of Bailey," ependymoblastomas and ependymogliomas, the latter "consisting of ependymal and astrocyte elements," already acknowledging the difficulties in defining a cell of origin versus dedifferentiation or even divergent differentiation in these tumors $[8,9]$.

Histologically, the main hallmarks of ependymoma are:

- Perivascular pseudo-rosettes, which consist of nucleus-free mantles, surrounded by a radial disposition of cells around blood vessels. These may be difficult to identify in lesions or areas with very high or very low cellularity;

- True ependymal rosettes and ependymal canals, composed of columnar cells arranged around a central lumen or cavity. These are very characteristic of ependymoma, but are not found in all cases;
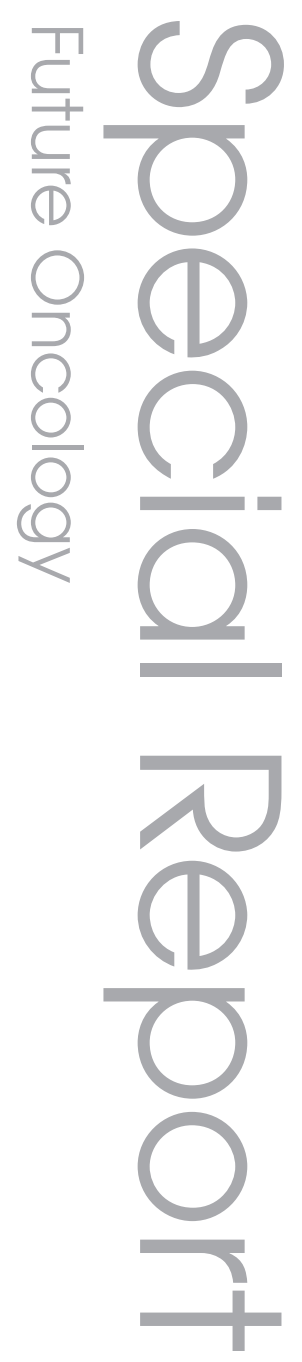

\section{Keywords}

- classification = ependyma rosette $=$ ependymal tumor - glial neoplasm = grading - predictive biomarker - tenascin-C

\section{Future $\because \cdots$ Medicine part of}


- Immunohistochemical stains in ependymoma generally show strong cytoplasmic positivity for glial fibrilary acidic protein, vimentin and CD56 [10]. Epithelial membrane antigen usually highlights the luminal surface of true rosettes and canals, and often shows intracytoplasmic dots [11]. OLIG2 is usually absent or stains only a few nuclei in ependymoma, and can be a useful marker in the differential diagnosis in favor of other glial tumors such as oligodendroglioma or pilocytic astrocytoma [10]. Its expression is more frequent in supratentorial ependymoma with simultaneous neuronal differentiation, which delineates a subgroup of patients that usually show a less aggressive course [12].

\section{Histological grading of ependymoma \& its prognostic impact}

According to the WHO classification and grading scheme for CNS tumors, ependymal tumors can be classified as follows:

- Subependymoma (WHO grade I), generally presenting in adults and associated with minimal morbidity;

- Myxopapillary ependymoma (WHO grade I), exclusively located in the spine and rarely seen in children with a known tendency for cerebrospinal fluid dissemination in this age group [13];

- Besides the classic histological form, different morphological variants are distinguished, which do not have prognostic implications per se (cellular, clear cell, tanicytic and papillary ependymoma). Intracranial pediatric ependymomas are mostly represented by either classic (WHO grade II) or anaplastic (WHO grade III) tumors [14,15].

The diagnosis of anaplastic ependymoma should be based on the presence of "increased cellularity, brisk mitotic activity, often associated with microvascular proliferation and pseudopalisading necrosis," criteria classically developed for astrocytic and oligodendroglial tumors. There is considerable histopathological variation among and within tumors, for instance, well demarcated nodules of densely cellular mitotically active cells can be found in approximately one-third of posterior fossa lesions [15], which results in great difficulty in grading them reliably [16-19]. Such difficulty is reflected by studies of clinically similar cohorts of children with intracranial ependymoma that report ratios of grade II to III tumors ranging between 17:1 and 1:7 - a striking discordance that could be attributed to various reasons such as intratumoral heterogeneity, interobserver variability, variations in definitions, the use of different histological criteria, the uneven application of criteria for anaplasia by review pathologists, and idiosyncratic small patient cohorts, as has been pointed out in excellent works dealing with this subject $[14,15]$. Moreover, attempts from efforts by groups of neuropathologists with experience in pediatric brain tumors have not yet validated an alternative classification scheme that would be easily reproducible [14-16]. More importantly, among pediatric ependymomas in particular, it is not clear whether lesions classified as grade II or III, according to either WHO classification schemes or alternative grading systems, have a clearly distinct outcome [14,20,21]. Posterior fossa ependymomas are treated with adjuvant radiotherapy, whatever their grade, while some cooperative groups, especially in the USA, consider that grade II supratentorial ependymomas can be observed after complete resection. Although anaplastic variants are regarded as being more aggressive and treated with adjuvant therapy following surgery, grading in its present form needs to be more robustly validated through large cooperative multi-institutional trials.

\section{Molecular classification of ependymomas \& its potential prognostic impact}

In the 2007 WHO classification, where tumors are diagnosed according to the appearance of the main cellular component, ependymomas are neoplasms resembling the ependymal cells and are, therefore, thought to originate from the ependymal layer of the ventricular system. However, characterization of tumor stem cells in ependymoma points to an origin from radial glia cells $[22,23]$. Indeed, these tumorinitiating cells harbor specific markers of radial glial progenitor cells such as BLBP or RC2 [22]. Ependymoma stem cells show Notch pathway activation (Figure 1). Numerous studies have highlighted that location was a strong driver for the biology of these neoplasms [22-26]. Intracranial ependymomas have, therefore, been separated into subclasses according to their chromosomal imbalances [23,27], differentiation [5,12] or driver mutation $[23,28,29]$. The exact number of molecular subclasses of ependymomas is not completely fixed, but there are at least two posterior fossa subtypes [5] and two supratentorial subtypes [12] 
described. Some authors, considering both driver mutations and chromosomal abnormalities, have counted up to nine different subclasses of ependymomas [23]. Supratentorial ependymomas segregate into two different prognostic groups according to the presence of neuronal differentiation; the presence of $N F L 70$ expression is associated with a better progression-free survival at 3 years ( 90 vs $28 \%$; $=0.007 \log$-rank test) and overall survival at 5 years ( 90 vs $60 \% ; p=0.14$ log-rank test) irrespective of the other known risk factors [12]. Infratentorial ependymomas segregate into two different prognostic groups according to their gene expression profiles [5]; group $\mathrm{A}$ is characterized by a more adverse outcome, more frequent location in the cerebellopontine angle and cerebellar invasion, younger age, overexpression of merosin/laminin- $\alpha 2$ (LAMA2) or tenascin-C (TNC); while group $\mathrm{B}$ comprises intraventricular and intramedullary tumors in adolescent/adult patients with overexpression of NELL2 and genes involved in ciliogenesis or microtubule assembly. The 5-year progression-free and overall survival was significantly worse in group A ( 44 vs $75 \% ; \mathrm{p}=0.017$ and 65 vs $95 \% ; \mathrm{p}=0.0048$ ).

Microarray studies are able to show clear differences among subgroups of ependymomas according to location, but these subgroups may also have some biological features in common in addition to histology, such as pathways associated with resistance to therapy. Indeed, comparing recurrences with initial tumors showed the frequent overexpression of kinetochore proteins and stem cell markers, as well as downregulation of metallothioneins [6]. In addition, hypomethylation of specific loci, as well as global hypomethylation, was recently shown to occur more frequently in aggressive and recurrent ependymomas, irrespective of their location [30]. Of note, the groups with the best prognosis show neuronal differentiation markers in both locations (NELL2 in the posterior fossa and NFL7O in the supratentorial region). One should, therefore, consider studying prognostic markers, both according to and irrespective of the location of the tumor.

Another approach for prognostication with biological markers could be to use driving mutations to define subclasses of tumors. Except for neurofibromatosis type 2, where ependymoma tend to be confined to the spinal cord, no real predisposition syndrome has been linked to ependymoma and most cases are sporadic. Awaiting for the results of the whole genome sequencing of ependymomas, only few potential

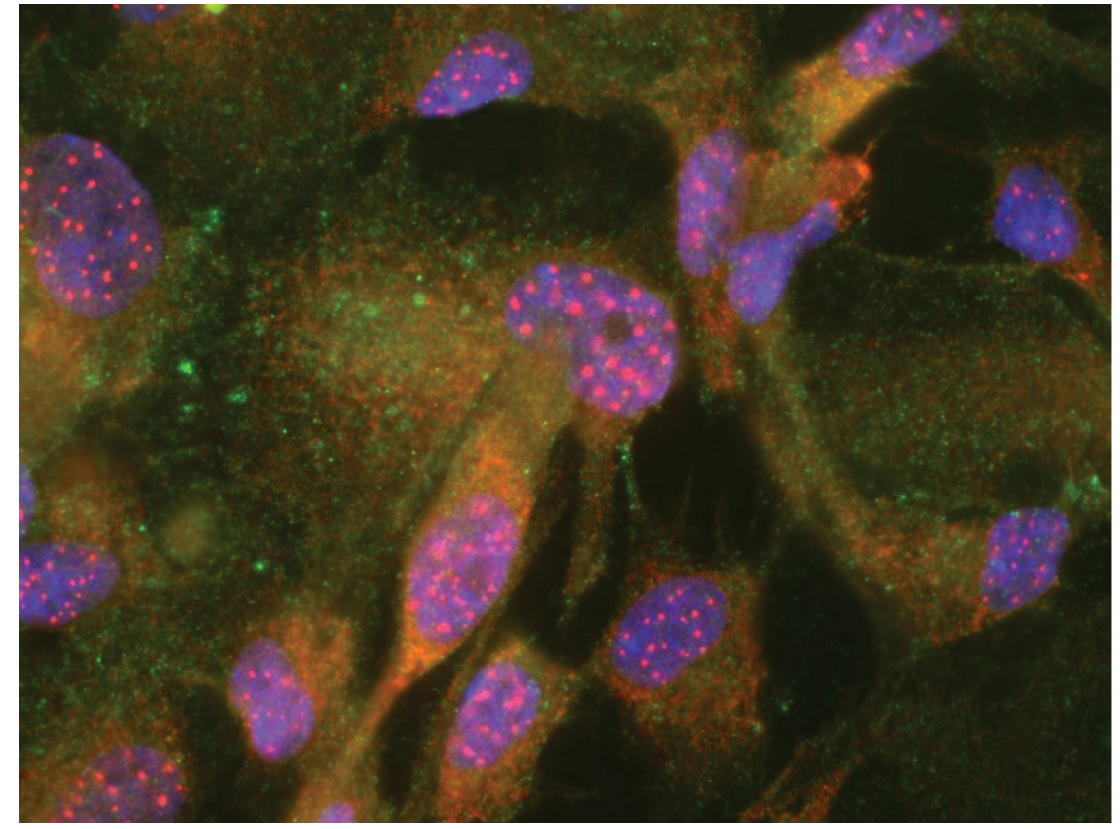

Figure 1. Activation of the Notch pathway in adherent ependymoma stem cell cultures. Immunohistochemistry for HES-1 shows intranuclear dots of the major effector of the Notch pathway.

drivers of ependymoma oncogenesis have been identified, namely Notch-1 gain/mutation [28], EPHB2 amplification [23] and Evi-1 overexpression [31]. This latter one was considered as a possible adverse prognostic factor in one retrospective study and would need further validation.

\section{Prognostic impact of chromosomal imbalances}

Differences between ependymomas in children and adults have been constantly reported. Besides different preferential location (supratentorial and spinal in adults, posterior fossa in children), ependymomas in children have largely been shown to have a worse prognosis. A comprehensive meta-analysis of 13 comparative genomic hybridization (CGH) studies and literature review on biomarkers has shown striking differences between pediatric and adult ependymoma on a genetic level [32]. One of the most relevant difference with adult ependymomas is the higher frequency of 1q gain in pediatric tumors ( 20 vs $8 \% ; \mathrm{p}=0.0040$ ). This 1q gain has been linked to an adverse prognosis in several retrospective cohorts [27,28,33-35] and, more recently, in two infant and young children trial cohorts [36]. These studies used $\mathrm{CGH}$, array CGH or FISH using commercial probes for $1 \mathrm{q} 25$ or $1 \mathrm{q} 41$. While the prognostic value of this chromosomal aberration is undeniable, the best method for its detection has 
still to be defined. Indeed, up to $20 \%$ of cases can not be assessed by FISH on formalin-fixed paraffin-embedded. An alternative technique could be multiplex ligation-dependent probe amplification, which has recently been used in a German trial cohort where 1q status could be determined in almost $100 \%$ of cases [37].

Unlike adult cases, pediatric ependymomas more frequently display a balanced karyotype $[27,28,32,33]$, especially in infants and young children, who have the highest risk of recurrence. In a single center study, 9q33-34 gain (the locus of Notch1 and TNC) was the most frequent chromosomal aberration detected at recurrence and was associated with a worse outcome [28].

Three genomic risk groups that were recently identified and validated in mixed adult and pediatric cohorts were shown to improve prognostication over clinical risk stratification [27]; group 1 showed chromosome 9, 15q and 18 gains and chromosome 6 loss, and had the best prognosis; while group 3 exhibited chromosome $1 \mathrm{q}$ gain and 9p21.3 (CDKN2A/B) loss, and had the worse prognosis. An intermediate group 2 was characterized by a balanced CGH array profile. The usefulness of this classification needs to be validated in a prospective cohort and would require assessment on DNA, ideally extracted from frozen material.

\section{Microarray gene signature of poor prognosis}

In a recent study on adult and pediatric paraffin-embedded infratentorial ependymomas, a ten-gene signature was established that correlated with both worse overall survival and worse progression-free survival, and significantly correlated with a younger age at diagnosis [38]. Unsupervised clustering initially revealed two groups of tumors, one of them showing a signature similar to the group A described by Witt et al. [5]. This signature has not yet been validated and will require additional steps for standardization before it can be used for the prediction of recurrence.

\section{Proliferation markers as indicators of tumor aggressiveness}

Numerous studies have focused on the assessment of the proliferation index in pediatric ependymomas by immunohistochemistry for Ki67 and its relation to prognosis. Although most authors have shown an inverse and significant correlation between proliferation and prognosis $[16,39,40]$, other groups did not confirm this data [41,42]. All were retrospective studies, and for the great majority, the association was not confirmed in multivariate analysis. Location may be a confounding factor since supratentorial ependymoma usually have a higher proliferative index [12] despite often being associated with a better prognosis [43]. Moreover, the labeling index used as a cut-off in these various studies was extremely variable (ranging from 1 to $25 \%$ ), which renders definitive conclusions as to its prognostic value in pediatric ependymoma difficult. Interestingly, at recurrence, there is strong overexpression of genes involved in cell cycle progression such as Egr5 and ASPM and usually a higher proliferation rate [6]

Whether or not the proliferation index should be incorporated in ependymoma prognostication will need further studies and possibly its combination with other potential biological risk factors.

\section{Single molecular markers for prognosis}

Numerous studies have been performed to discover prognostic markers in this disease, based either on assimilation of known biomarkers in glioma or, more recently, on genomic or gene expression profiling (TABLE 1 ).

\section{Telomerase}

An immunohistochemical study on 65 pediatric ependymomas has shown human telomerase reverse transcriptase (hTERT) positivity in 58\% of cases. Multivariate analysis on this cohort identified hTERT as a potent prognostic predictor of survival in childhood ependymoma [44]. In a retrospective cohort of 83 pediatric patients with 133 ependymomas, including 31 at relapse, the same group showed that hTERT expression was correlated with proliferation index assessed by both MIB-1 and the mitotic count. This data suggested that hTERT could be a marker of aggressive behavior in these tumors [45]. However, it was subsequently shown that the antibody used in this study was, in fact, directed against nucleolin, a protein with the function of nuclear chaperone for hTERT [42]. This retrospective study of 97 intracranial tumors from 74 patients with pediatric intracranial ependymoma that evaluated several candidate prognostic markers, including nucleolin, KI67, members of the HER family and survivin, showed only low nucleolin expression ( $<50 \%$ cells) as a significant prognostic factor, with a favorable impact on overall survival and progression-free survival. In their series, patients with tumors with high immunohistochemical expression of 
Table 1. Biomarkers studied and their development status.

\begin{tabular}{|c|c|c|c|}
\hline Locus/gene investigated & Methodology & $\begin{array}{l}\text { Stage of development } \\
\text { of the biomarker }\end{array}$ & Ref. \\
\hline TERT/NCL & IHC, qRT-PCR & Discovery & {$[42,44,45]$} \\
\hline TOPO 2A & IHC, qRT-PCR & Discovery & [38] \\
\hline RTKs (HER3, EGFR, HER2/ERBB2) & IHC, qRT-PCR & Discovery & {$[42,47]$} \\
\hline TNC & IHC, qRT-PCR & Standardization & 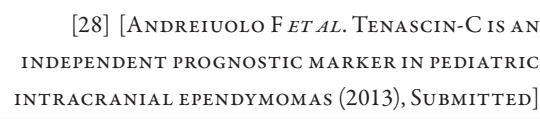 \\
\hline 1q gain & FISH, array CGH, MLPA & Confirmation & {$[32,35,37]$} \\
\hline 9q gain & FISH, array CGH & Discovery & [28] \\
\hline Nestin & $\mathrm{IHC}$ & Discovery & [51] \\
\hline NELL2/LAMA2 & $\mathrm{IHC}$ & Confirmation & [5] \\
\hline CGH class & Array CGH, FISH & Confirmation & [27] \\
\hline Neurofilament (NFP70) & $\mathrm{IHC}$ & Discovery & {$[12]$} \\
\hline EVI-1 & IHC, RT-PCR & Discovery & [31] \\
\hline TP53 & IHC & Discovery & [50] \\
\hline
\end{tabular}

nucleolin had a 5-year progression-free survival at 31 versus $74 \%$ for those with low expression. The unavailability of commercial antibodies (withdrawn from the market) and difficulties for establishing a threshold for positivity have considerably hampered the further utilization of this marker. Moreover, nucleolin could not be validated further as a prognostic marker in trial cohorts [Andreiuolo F ET AL. Tenascin-C IS AN INDEPENDENT PROGNOSTIC MARKER IN PEDIATRIC intRACRANiAl EPENDYMomas (2013), Submitted].

\section{EGF receptor}

The expression of specific growth factors, such as the EGFR, have been suggested as possible prognostic markers, both independently [34,46] or when coexpressed with caveolin-1 [47], but this has not been confirmed in further studies [42]. In myxopapillary ependymomas especially, EGFR expression could be a predictor of relapse [48].

\section{Intratumoral immune response}

As reported in other types of cancer, the immune system appears to influence the prognosis of childhood ependymoma. A recent study on 19 pediatric ependymomas showed the overexpression of immune function-associated genes with nonrecurrent lesions [49]. Among genes associated with both nonrecurrent phenotype and positively correlated with time to progression, $95 \%$ were associated with both innate and acquired immune function. In nonrecurrent lesions, the authors showed the overexpression of innate immune function genes in microglial cells and an increased number of CD4 lymphocytes, mediators of adaptative immune response, in nonrecurrent lesions. This interesting data should be confirmed on a larger series but points to an alternative direction in the study of prognostic factors in ependymoma. We have not found an increased number of CD3 positive cells in our trial series [ANDREIUOLO F ET AL. TENASCIN-C is AN INDEPENDENT PROgNostic MARKER IN PEDIATRIC INTRACRANIAL EPENDYMOMAS (2013), Submitted].

\section{Topoisomerase II}

The expression of topoisomerase II was linked to an adverse prognosis as part of the ten-gene signature described by Wani et al. [38]. Since robust antibodies exist, this biomarker is worth studying further, especially as it is linked to proliferation.

\section{TP53}

As a major cell-cycle regulator and guardian of the genome, $p 53$ has also been studied in ependymomas, where it is rarely mutated but occasionally overexpressed [17,50]. Its overexpression is linked to higher tumor grade, and eventually, worse prognosis, but its rare occurrence may preclude its use as a prognostic marker. 


\section{Nestin}

Tumor stem cells have been suggested to play an important role in tumor resistance to irradiation or chemotherapy. Milde et al. have recently shown that nestin expression could serve as an adverse prognostic factor [51]. Confirmatory studies are warranted, but its frequent overexpression in ependymomas may preclude its usefulness as biomarker.

\section{Tenascin-C}

A candidate gene strategy exploring the 9q33-34 gain discovered TNC as significantly overexpressed both at the mRNA and protein levels [28]. TNC is a direct target of Notch-1, whose pathway is usually activated in ependymoma through various mechanisms (Figure 2). It may constitute a major component of the extracellular matrix of the tumor stem cell niche. Its prognostic impact was explored on four different trial cohorts. Its overexpression shown by immunohistochemistry was associated with a 1.7-fold increase in relapse or death in multivariate analysis [Andreivolo F ET AL. Tenascin-C is An INDEPENDENT PROGNOSTIC MARKER IN PEDIATRIC INTRACRANIAL ependymomas (2013), Submitted]. This effect on survival was further confirmed in two independent cohorts. This biomarker was shown to be robust and reproducible, and prospective validation is warranted.

\section{Future perspective: not one, but a combination of biomarkers}

It is unlikely that one single biomarker could describe the complex biology of ependymomas. For example, 1q gain or p53 overexpression are

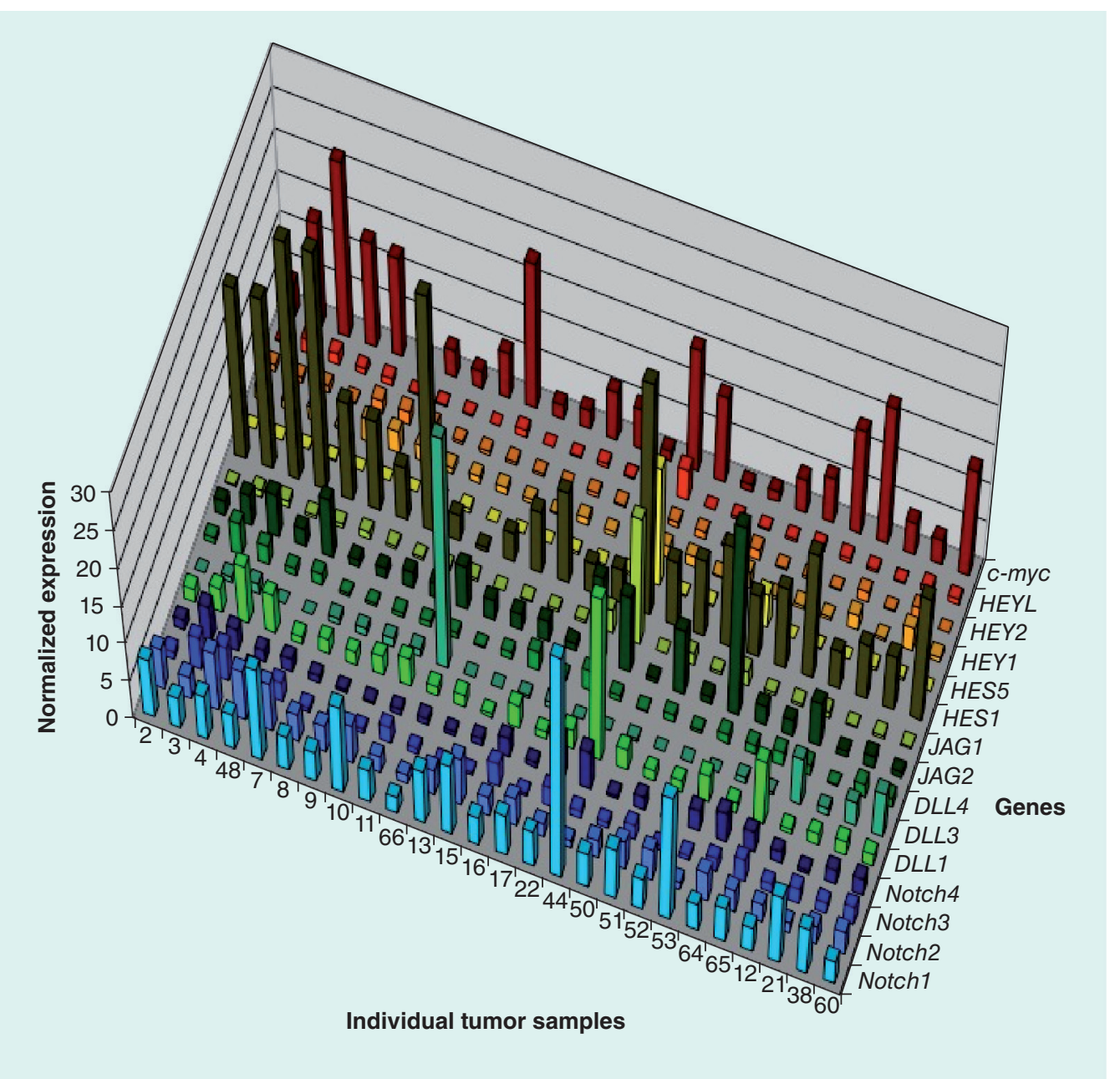

Figure 2. Notch pathway in ependymoma primary tumors. Expression of Notch pathway receptors (1-4), ligands (DLL1, 3 and 4, and JAG1 and 2) and major effectors (HES1 and 5, and HEY1 and 2; HEYL and c-myc) were quantified with reverse-transcription PCR in pediatric primary ependymoma tumor samples. Baseline level corresponds to the normal brain. Genes significantly overexpressed compared with baseline are: Notch1, DLL1, JAG1, HES1 and c-myc. A total of $80 \%$ of the samples had the overexpression of at least the combination of one receptor (most frequently Notch1), one ligand (most frequently JAG1) and one effector (most frequently HES1). 
Table 2. Comparison of adult and pediatric ependymomas with respect to biomarkers for poor prognosis.

\begin{tabular}{|lll|}
\hline Biomarkers & Adult & Child \\
\hline WHO grade III & Yes & Discussed \\
\hline 1q gain $\pm 9 p 21.3$ loss & Yes (but rare) & Yes \\
\hline 9q33-34 gain & No & $\begin{array}{l}\text { To be confirmed (posterior fossa } \\
\text { tumors) }\end{array}$ \\
\hline EGFR overexpression & Yes & To be confirmed \\
NFL70 expression & Not tested & To be confirmed (supratentorial) \\
\hline TNC expression & Not tested & Young children with posterior \\
& & fossa tumors \\
hTERT/NCL & Not tested & Discussed \\
\hline EVI-1 expression & Yes (posterior fossa) & Yes (posterior fossa) \\
\hline
\end{tabular}

only present in a small subset of ependymomas. One would probably need a set of biomarkers defining a composite biological risk score, eventually specific to a given location or age (TABLE 2 ). Moreover, if functional subgroups of ependymomas are identified as in medulloblastoma, prognostic biomarkers' refinement would eventually need to be limited to each subgroup. In addition, it would be useful to define predictive biomarkers for the response to specific treatments such as chemotherapy. Due to the rarity of the disease and its biological complexity, international cooperation is highly desirable to ensure that robust and reliable diagnostic and prognostic biomarkers are validated in the near future. The establishment of reliable biomarkers will need to go through a systematic process including the standardization of the assay and its validation in routine conditions within a prospective trial. Similarly to medulloblastoma, we will be able to define high-risk biological profiles that could potentially lead to significant treatment changes.

\footnotetext{
Financial \& competing interests disclosure

The ependymoma research project at the Gustave

Roussy Institute is supported by the following charities:

Etoile de Martin, Amélie La Vie and Association pour

la Recherche sur les Tumeurs Cérébrales, Enfants et

Santé and Société Française des Cancers de l'Enfant,

Léa Princesse Eternelle, and Timéo. The Institut

National du Cancer supported the PhD work of

$F$ Andreiuolo. Research funding was obtained for drug

development in ependymoma from Hoffmann-La Roche.

The authors have no other relevant affliations or finan-

cial involvement with any organization or entity with

a financial interest in or financial conflict with the

subject matter or materials discussed in the manuscript

apart from those disclosed.

No writing assistance was utilized in the production of this manuscript.
}

\section{Executive summary}

\section{Grading of ependymoma is still a controversial issue}

- Ependymoma of a higher grade tend to have a worse prognosis, especially in the posterior fossa.

- Grading according to the current WHO grading is not reproducible enough among pathologists to ensure reliable patient stratification in trials.

There is no real consensus on a given molecular classification of ependymoma, unlike in medulloblastoma

- Location defines specific gene expression signature and genomic abnormalities.

- Many driver molecular abnormalities have yet to be identified in these tumors besides the few described to date (e.g., Notch-1 gain/mutations, EPHB2 amplification).

\section{Prognostic biomarkers}

- None of the current biomarkers has been completely validated yet.

- Several prognostic markers, such as 1q gain, EGFR, TNC or hTERT-nucleolin overexpression, warrant further prospective studies.

- Standardization of the assays is critical before these biomarkers can be exploited for patient stratification in trials.

\section{Conclusion}

- We recognize that ependymomas can no longer be considered a single tumor entity and that biomarkers need to be studied according to several clinical characteristics such as age (children vs adults) and location (spine, posterior fossa or supratentorial region). 


\section{References}

Papers of special note have been highlighted as:

- of interest

- of considerable interest

1. Zulch KJ. Brain Tumors. Their Biology and Pathology. Springer-Verlag, NY, USA (1986).

2. Störch E. Uber die pathologish-anatomischen Vorgänge am Stützgerüst des

Zentralnervensystems. Virchow Arch. 157, 127-171 (1899).

3. Mallory FB. Three gliomata of ependymal origin; two in the fourth ventricle, one subcutaneous over the coccyx. J. Med. Res. 8, 1-10.1 (1902).

4. Kobayashi T, Dynlacht BD. Regulating the transition from centriole to basal body. J. Cell Biol. 193, 435-444 (2011).

5. Witt H, Mack SC, Ryzhova M et al. Delineation of two clinically and molecularly distinct subgroups of posterior fossa ependymoma. Cancer Cell 20, 143-157 (2011).

- Large gene expression microarray study in posterior fossa ependymoma that highlights the adverse prognosis of mesenchymal transition.

6. Peyre M, Commo F, Dantas-Barbosa C et al. Portrait of ependymoma recurrence in children: biomarkers of tumor progression identified by dual-color microarray-based gene expression analysis. PLoS One 5, e12932 (2010).

7. Judkins AR, Ellison DW. Ependymoblastoma: dear, damned, distracting diagnosis, farewell! Brain Pathol. 20, 133-139 (2010).

8. Roussy G, Lhermitte J, Cornil L. Essai de classification des tumeurs cérébrales. Ann. Anat. Pathol. 1, 333-378 (1924).

9. Roussy G, Oberling C. Histologic classification of tumors of the central nervous system. Arch. Neurol. Psychiatry 27, 1281-1289 (1932).

10. Godfraind C. Classification and controversies in pathology of ependymomas. Childs Nerv. Syst. 25, 1185-1193 (2009).

11. Hasselblatt M, Paulus W. Sensitivity and specificity of epithelial membrane antigen staining patterns in ependymomas. Acta Neuropathol. 106, 385-388 (2003).

12. Andreiuolo F, Puget S, Peyre M et al. Neuronal differentiation distinguishes supratentorial and infratentorial childhood ependymomas. Neuro. Oncol. 12(11), 1126-1134 (2010).

- First study in pediatric supratentorial ependymomas that describe the better outcome of tumors with neuronal differentiation.
13. Fassett DR, Pingree J, Kestle JR. The high incidence of tumor dissemination in myxopapillary ependymoma in pediatric patients. Report of five cases and review of the literature. J. Neurosurg. 102, 59-64 (2005).

14. Ellison DW, Kocak M, Figarella-Branger D et al. Histopathological grading of pediatric ependymoma: reproducibility and clinical relevance in European trial cohorts. J. Negat. Results Biomed. 10, 7 (2011).

- This European initiative to unify the criteria for grading of ependymoma is proposing a new scheme that was shown to be more reproducible.

15. Tihan T, Zhou T, Holmes E, Burger PC, Ozuysal S, Rushing EJ. The prognostic value of histological grading of posterior fossa ependymomas in children: a Children's Oncology Group study and a review of prognostic factors. Mod. Pathol. 21, 165-177 (2008).

16. Figarella-Branger D, Civatte M, BouvierLabit $\mathrm{C}$ et al. Prognostic factors in intracranial ependymomas in children. J. Neurosurg. 93, 605-613 (2000).

17. Korshunov A, Golanov A, Timirgaz V. Immunohistochemical markers for prognosis of ependymal neoplasms. J. Neurooncol. 58, 255-270 (2002).

18. Robertson PL, Zeltzer PM, Boyett JM et al. Survival and prognostic factors following radiation therapy and chemotherapy for ependymomas in children: a report of the Children's Cancer Group. J. Neurosurg. 88, 695-703 (1998).

19. Pollack IF, Gerszten PC, Martinez AJ et al. Intracranial ependymomas of childhood: long-term outcome and prognostic factors. Neurosurgery 37, 655-666; discussion 666-657 (1995).

20. McGuire CS, Sainani KL, Fisher PG. Both location and age predict survival in ependymoma: a SEER study. Pediatr. Blood Cancer 52, 65-69 (2009).

21. Massimino M, Buttarelli FR, Antonelli M, Gandola L, Modena P, Giangaspero, F. Intracranial ependymoma: factors affecting outcome. Future Oncol. 5, 207-216 (2009).

22. Taylor MD, Poppleton H, Fuller C et al. Radial glia cells are candidate stem cells of ependymoma. Cancer Cell 8, 323-335 (2005).

- Points out that the oncogenesis is dependent on the location of the tumor and ependymomas can derive from radial glial cells.

23. Johnson RA, Wright KD, Poppleton $\mathrm{H}$ et al. Cross-species genomics matches driver mutations and cell compartments to model ependymoma. Nature 466, 632-636. (2010).
24. Modena P, Lualdi E, Facchinetti F et al. Identification of tumor-specific molecular signatures in intracranial ependymoma and association with clinical characteristics. J. Clin. Oncol. 24, 5223-5233 (2006).

25. Palm T, Figarella-Branger D, Chapon F et al. Expression profiling of ependymomas unravels localization and tumor gradespecific tumorigenesis. Cancer 115, 3955-3968 (2009).

26. Schneider D, Monoranu CM, Huang B et al. Pediatric supratentorial ependymomas show more frequent deletions on chromosome 9 than infratentorial ependymomas: a microsatellite analysis. Cancer Genet. Cytogenet. 191, 90-96 (2009).

27. Korshunov A, Witt H, Hielscher T et al. Molecular staging of intracranial ependymoma in children and adults. J. Clin. Oncol. 28, 3182-3190 (2010).

- Large genomic study in pediatric and adult ependymomas showing that specific chromosomal imbalances may drive more adverse disease course.

28. Puget S, Grill J, Valent A et al. Candidate genes on chromosome 9q33-34 involved in the progression of childhood ependymomas. J. Clin. Oncol. 27, 1884-1892 (2009).

- Describes two new genes, Notch1 and $T N C$, involved in ependymoma oncogenesis that are preferentially gained in recurrent ependymomas, the former also being mutated in a subset of tumors.

29. Gottardo NG, Poppleton H, Johnson R et al. Concurrent activation of Notch cell signaling and deletion of Ink4/Arf in radial glia causes cerebral ependymoma. Program and Abstracts of the 13th International Symposium on Pediatric Neuro-Oncology. Chicago, IL, USA, 29 June-2 July 2008 (Abstract BIO6).

30. Xie H, Wang M, De F. Bonaldo M et al. Epigenomic analysis of Alu repeats in human ependymomas. Proc. Natl Acad. Sci. USA 107(15), 6952-6957 (2010).

31. Koos B, Bender $\mathrm{S}$, Witt $\mathrm{H}$ et al. The transcription factor evi-1 is overexpressed, promotes proliferation, and is prognostically unfavorable in infratentorial ependymomas. Clin. Cancer Res. 17, 3631-3637 (2011).

32. Kilday JP, Rahman R, Dyer S et al. Pediatric ependymoma: biological perspectives. Mol. Cancer Res. 7, 765-786 (2009).

33. Dyer S, Prebble E, Davison Vet al. Genomic imbalances in pediatric intracranial ependymomas define clinically relevant groups. Am. J. Pathol. 161, 2133-2141 (2002). 
34. Mendrzyk F, Korshunov A, Benner A et al. Identification of gains on $1 \mathrm{q}$ and epidermal growth factor receptor overexpression as independent prognostic markers in intracranial ependymoma. Clin. Cancer Res. 12, 2070-2079 (2006).

35. Godfraind C, Kaczmarska JM, Kocak M et al. Distinct disease-risk groups in pediatric supratentorial and posterior fossa ependymomas. Acta Neuropathol. 124(2), 247-257 (2012).

36. Kilday JP, Mitra B, Domerg C et al. Copy number gain of $1 \mathrm{q} 25$ predicts poor progression-free survival for pediatric intracranial ependymomas and enables patient risk stratification. Clin. Cancer Res. 18, 2001-2011 (2002).

37. Velez-Char N, Doerner E, zur Muehlen A et al. Analysis of chromosome 1q gain as genetic marker for risk stratification of pediatric ependymoma patients - validation as an adverse prognostic marker in the German multicenter HIT 2000 trial. Program and Abstracts of the 15th International Symposium on Pediatric Neuro-Oncology. Toronto, ON, Canada, 24-27 June 2012 (Abstract EM23).

38. Wani K, Armstrong TS, Vera-Bolanos E et al. A prognostic gene expression signature in infratentorial ependymoma. Acta Neuropathol. 123, 727-738 (2012).

39. Preusser M, Heinzl H, Gelpi E et al. Ki67 index in intracranial ependymoma: a promising histopathological candidate biomarker. Histopathology 53, 39-47 (2008).

40. Zamecnik J, Snuderl M, Eckschlager T et al. Pediatric intracranial ependymomas: prognostic relevance of histological, immunohistochemical, and flow cytometric factors. Mod. Pathol. 16, 980-991 (2003).

41. Prayson RA. Clinicopathologic study of 61 patients with ependymoma including MIB-1 immunohistochemistry. Ann. Diagn. Pathol. 3, 11-18 (1999).

42. Ridley L, Rahman R, Brundler MA et al. Multifactorial analysis of predictors of outcome in pediatric intracranial ependymoma. Neuro. Oncol. 10, 675-689 (2008).

43. Grill J, Le Deley MC, Gambarelli D et al. Postoperative chemotherapy without irradiation for ependymoma in children under 5 years of age: a multicenter trial of the French Society of Pediatric Oncology. J. Clin. Oncol. 19, 1288-1296 (2001).

44. Tabori U, Ma J, Carter M et al. Human telomere reverse transcriptase expression predicts progression and survival in pediatric intracranial ependymoma. J. Clin. Oncol. 24, 1522-1528 (2006).

45. Tabori U, Wong V, Ma J et al. (2008). Telomere maintenance and dysfunction predict recurrence in paediatric ependymoma. Br. J. Cancer 99, 1129-1135 (2008).
46. Gilbertson RJ, Bentley L, Hernan R et al. ERBB receptor signaling promotes ependymoma cell proliferation and represents a potential novel therapeutic target for this disease. Clin. Cancer Res. 8, 3054-3064 (2002).

47. Senetta R, Miracco C, Lanzafame $S$ et al. Epidermal growth factor receptor and caveolin-1 coexpression identifies adult supratentorial ependymomas with rapid unfavorable outcomes. Neuro. Oncol. 13(2), 176-183 (2011).

48. Verma A, Zhou H, Chin S, Bruggers C, Kestle J, Khatua, S. EGFR as a predictor of relapse in myxopapillary ependymoma. Pediatr. Blood Cancer 59(4), 746-748 (2012).

49. Donson AM, Birks DK, Barton VN et al. Immune gene and cell enrichment is associated with a good prognosis in ependymoma. J. Immunol. 183, 7428-7440 (2009).

50. Sharma MC, Ghara N, Jain D, Sarkar C, Singh M, Mehta VS. A study of proliferative markers and tumor suppressor gene proteins in different grades of ependymomas. Neuropathology 29(2), 148-155 (2009).

51. Milde T, Hielscher T, Witt H et al. Nestin expression identifies ependymoma patients with poor outcome. Brain Pathol. 22(6), 848-860 (2012). 